

\title{
A NONLINEAR TIMOSHENKO BEAM FORMULATION BASED ON STRAIN GRADIENT THEORY
}

\author{
Reza Ansari, Raheb Gholami And Mohammad Ali Darabi
}

\begin{abstract}
Developed herein is a comprehensive geometrically nonlinear size-dependent microscale Timoshenko beam model based on strain gradient and von Kármán theories. The nonlinear governing equations and the corresponding boundary conditions are derived from employing Hamilton's principle. A simply supported microbeam is considered to delineate the nonlinear size-dependent free vibration behavior of the presented model. Utilizing the harmonic balance method, the solution for free vibration is presented analytically. The influence of the geometric parameters, Poisson's ratio, and material length-scale parameters on the linear frequency and nonlinear frequency ratio are thoroughly investigated. The results obtained from the present model are compared, in special cases, with those of the linear strain gradient theory, linear and nonlinear modified couple stress theory, and linear and nonlinear classical models; excellent agreement is found. It is concluded that the nonlinear natural frequency and nonlinear frequency ratio predicted by strain gradient theory are more precise than those from the other theories mentioned, especially for shorter beams.
\end{abstract}

\section{Introduction}

Micro- and nanoscale beams are increasingly used in micro- and nanoelectromechanical systems such as vibration shock sensors [Lun et al. 2006], electrostatically excited microactuators [Moghimi Zand and Ahmadian 2009; Mojahedi et al. 2010], microswitches [Coutu et al. 2004], and atomic force microscopes [Mahdavi et al. 2008]. The thickness of microscale beams is on the order of microns and submicrons. The size-dependent deformation behavior in microscale beams has been detected experimentally [Fleck et al. 1994; Lam et al. 2003]. Moreover, experiments confirm the significance of the effects of nonlinearity on the behavior of micro and nanomechanical resonators [Turner et al. 1998; Craighead 2000; Scheible et al. 2002].

Due to the absence of a material length-scale parameter, classical continuum mechanics is not able to interpret and predict such a size-dependent phenomenon which occurs in micron and submicron-scale structures. Accordingly, many attempts have been made to develop different size-dependent continuum theories such as the couple stress elasticity [Mindlin and Tiersten 1962; Toupin 1962], nonlocal elasticity [Eringen 1972], strain gradient elasticity [Aifantis 1999], and surface elasticity [Gurtin et al. 1998] theories. On the basis of elasticity theory, Yang et al. [2002] proposed a modified couple stress theory for elasticity in which only one higher-order material constant exists in the constitutive equations. In recent years, this theory has been applied by many researchers to interpret the size-dependent static and

Keywords: microbeams, strain gradient elasticity, modified couple stress theory, size effect, nonlinear behavior, Timoshenko beam theory. 
vibration behaviors of microstructures [Lazopoulos 2004; Park and Gao 2006; Kong et al. 2008; Ma et al. 2008; 2010; 2011; Asghari et al. 2010b; 2010a; Lazopoulos and Lazopoulos 2010].

Lazopoulos [2004] was apparently the first to employ the von Kármán kinematic relations in nonclassical analyses of beams and plates. Mindlin [1965] proposed a higher-order gradient theory for elastic materials by considering the first and second derivatives of the strain tensor effective on the strain energy density. In [Fleck and Hutchinson 1993; 1997; 2001], Mindlin's proposition was reformulated by considering only the first derivative of the strain this was called strain gradient theory. In comparison to couple stress theory, this theory comprises several higher-order stress components together with the classical and couple stresses. This means that couple stress theory can be attained via strain gradient theory by omitting the additional higher-order stress components. In this direction, Lam et al. [2003] modified strain gradient theory by considering three higher-order material constants in the constitutive equations. Also, this theory can be regenerated into the modified couple stress theory of [Yang et al. 2002] by setting the two material length-scale parameters equal to zero. In another work, Ma et al. [2011] developed a nonclassical Mindlin plate model based on modified couple stress theory. Tsepoura et al. [2002] have investigated the size effect of the Bernoulli-Euler beam model. In [Papargyri-Beskou et al. 2003b; Park and Gao 2006], based on the gradient elasticity theory with surface energy of [Vardoulakis and Sulem 1995], a higher-order Bernoulli-Euler beam model was expanded. Also, a cantilever beam subjected to bending and a cracked bar under stretching have been investigated by using the strain gradient beam model of [Giannakopoulos and Stamoulis 2007]. Wang et al. [2010], on the basis of strain gradient elasticity theory, developed a microscale Timoshenko beam model in which the Poisson effect was incorporated and also investigated the static bending and free vibration of a simply supported microscale Timoshenko beam to illustrate this model. Kahrobaiyan et al. [2011] developed a nonlinear size-dependent Euler-Bernoulli beam model based on strain gradient theory.

In the current work, by employing strain gradient theory and Hamilton's principle, a large-deformation size-dependent Timoshenko microbeam model is presented. The present model accommodates some previously published beam models including the linear couple stress [Park and Gao 2006; Kong et al. 2008; Ma et al. 2008], linear strain gradient [Kaneko 1975; Kahrobaiyan et al. 2011], nonlinear couple stress [Asghari et al. 2010b], nonlinear strain gradient [Kahrobaiyan et al. 2011] theories. Then, utilizing this model, the nonlinear size-dependent free vibration behavior of simply supported microbeams is described using the harmonic balance method. The numerical results are illustrated to study the effects of the geometric parameters, Poisson's ratio, and material length-scale parameters on the linear frequency and nonlinear frequency ratio.

\section{Preliminaries}

In comparison to the modified couple stress theory of [Yang et al. 2002], Lam et al. [2003] has presented a strain gradient elasticity theory which in addition to the symmetric rotation gradient tensor presents two additional gradient tensors, the dilatation gradient tensor and the deviatoric stretch gradient tensor. These tensors are specified by two classical material constants for isotropic linear elastic materials and three independent material length-scale parameters. The stored strain energy $U_{m}$ in a continuum constructed by a linear elastic material occupying a region $\Omega$ with infinitesimal deformations is given by

$$
U_{m}=\frac{1}{2} \int_{\Omega}\left(\sigma_{i j} \varepsilon_{i j}+p_{i} \gamma_{i}+\tau_{i j k}^{(1)} \eta_{i j k}^{(1)}+m_{i j}^{s} \chi_{i j}^{s}\right) d v
$$


in which the components of the strain tensor, the dilatation gradient tensor, the deviatoric stretch gradient tensor, and the symmetric rotation gradient tensor, respectively represented by $\varepsilon_{i j}, \gamma_{i}, \eta_{i j k}^{(1)}$, and $\chi_{i j}^{s}$ [Lam et al. 2003], are defined as

$$
\begin{gathered}
\varepsilon_{i j}=\frac{1}{2}\left(u_{i, j}+u_{j, i}\right), \quad \gamma_{i}=\varepsilon_{m m, i}, \\
\eta_{i j k}^{(1)}=\frac{1}{3}\left(\varepsilon_{j k, i}+\varepsilon_{k i, j}+\varepsilon_{i j, k}\right)-\frac{1}{15} \delta_{i j}\left(\varepsilon_{m m, k}+2 \varepsilon_{m k, m}\right)-\frac{1}{15}\left[\delta_{j k}\left(\varepsilon_{m m, i}+2 \varepsilon_{m i, m}\right)+\delta_{k i}\left(\varepsilon_{m m, j}+2 \varepsilon_{m j, m}\right)\right], \\
\chi_{i j}^{s}=\frac{1}{2}\left(\theta_{i, j}+\theta_{j, i}\right), \quad \theta_{i}=\frac{1}{2}(\operatorname{curl}(u))_{i},
\end{gathered}
$$

where $u_{i}$ represents the components of the displacement vector $u, \theta_{i}$ is the infinitesimal rotation vector $\theta$, and $\delta$ is Kronecker's delta. For a linear isotropic elastic material, the stresses are explained by the kinematic parameters effective on the strain energy density which are given in the following constitutive relations [Lam et al. 2003]:

$$
\sigma_{i j}=\lambda \operatorname{tr}(\varepsilon) \delta_{i j}+2 \mu \varepsilon_{i j}, \quad p_{i}=2 \mu l_{0}^{2} \gamma_{i}, \quad \tau_{i j k}^{(1)}=2 \mu l_{1}^{2} \eta_{i j k}^{(1)}, \quad m_{i j}^{s}=2 \mu l_{2}^{2} \chi_{i j}^{s} .
$$

The parameters $\boldsymbol{p}, \boldsymbol{\tau}^{(\mathbf{1})}$, and $\boldsymbol{m}^{s}$ are called the higher-order stresses. In the constitutive equation of the classical stress $\sigma$, the parameters $\lambda$ and $\mu$ are the bulk and shear modules, respectively, and are given as [Timoshenko and Goodier 1970]

$$
\lambda=\frac{E v}{(1+v)(1-2 v)}, \quad \mu=\frac{E}{2(1+v)} .
$$

Also, $l_{0}, l_{1}$, and $l_{2}$ appearing in the higher-order stresses represent the additional independent material length-scale parameters connected with the dilatation gradients, deviatoric stretch gradients, and symmetric rotation gradients, respectively. The kinematics of the Timoshenko beam theory can be written as [Reddy 2007]

$$
u_{1}=u(x, t)-z \psi(x, t), \quad u_{2}=0, \quad u_{3}=w(x, t),
$$

where $u(x, t), w(x, t)$, and $\psi(x, t)$ stand for the axial displacement of the center of sections, the lateral deflection of the beam, and the rotation angle of the cross section with respect to the vertical direction.

\section{Nonlinear formulation of motion and corresponding boundary conditions}

As depicted in Figure 1, a straight beam of length $L$ subjected to an initial axial load $N_{0}(x)$ is considered in which its top and bottom surfaces are perpendicular to the $z$-direction. The centroid of each section is assumed to be located on the plane $z=0$. The kinematic parameters, boundary conditions, and loading of a Timoshenko beam modeled based on the strain gradient theory are demonstrated in this figure. In addition, $G(x, t)$ is related to the axial body force per unit length and $F(x, t)$ denotes the transverse distributed force intensity per unit length.

Based on Timoshenko beam theory under the assumption of small strains and moderate rotations, the nonlinear strain-displacement relations of a beam subjected to large-amplitude vibrations can be approximated by the von Kármán relation as [Reddy 2003; Ma et al. 2008; Asghari et al. 2010b]

$$
\varepsilon_{11}=\frac{\partial u_{1}}{\partial x}+\frac{1}{2}\left(\frac{\partial w}{\partial x}\right)^{2}=\frac{\partial u}{\partial x}-z \frac{\partial \psi}{\partial x}+\frac{1}{2}\left(\frac{\partial w}{\partial x}\right)^{2}, \quad \varepsilon_{13}=\frac{1}{2}\left(\frac{\partial w}{\partial x}-\psi\right) .
$$



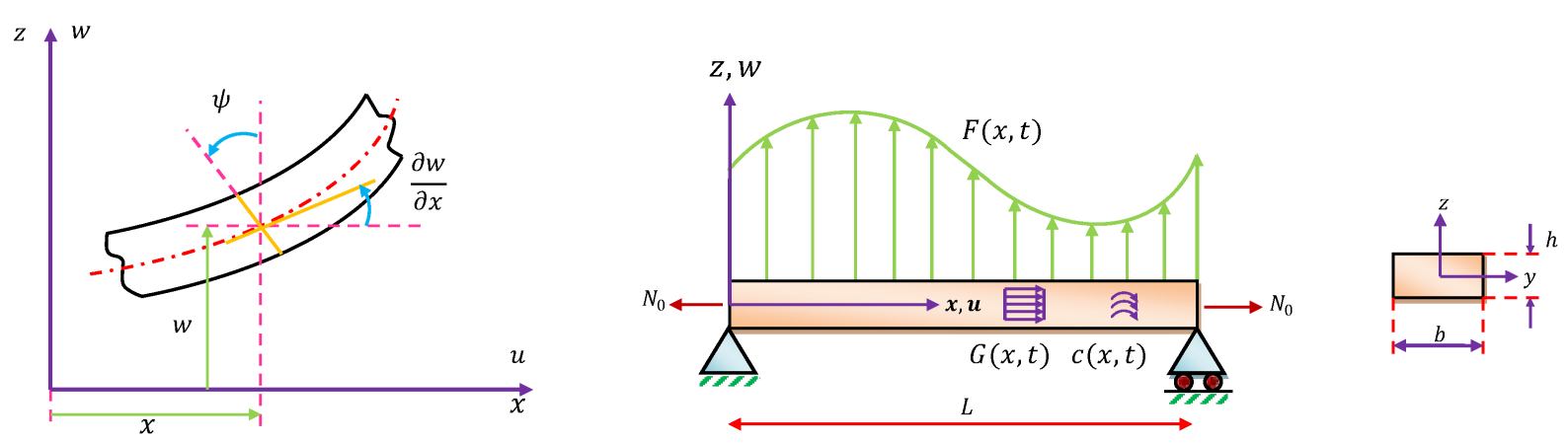

Figure 1. Schematic of a simply supported Timoshenko beam: kinematic parameters, coordinate system, geometry, and loading.

By inserting (5) and (6) into (2), the following nonzero components of $\boldsymbol{\theta}, \boldsymbol{\chi}^{\boldsymbol{s}}, \boldsymbol{\gamma}$, and $\boldsymbol{\eta}^{(\mathbf{1})}$ will be achieved:

$$
\begin{gathered}
\theta_{2}=-\frac{1}{2}\left(\psi+\frac{\partial w}{\partial x}\right), \quad \chi_{12}^{s}=\chi_{21}^{s}=-\frac{1}{4}\left(\frac{\partial \psi}{\partial x}+\frac{\partial^{2} w}{\partial x^{2}}\right), \\
\gamma_{1}=\frac{\partial^{2} u}{\partial x^{2}}-z \frac{\partial^{2} \psi}{\partial x^{2}}+\frac{\partial w}{\partial x} \cdot \frac{\partial^{2} w}{\partial x^{2}}, \quad \gamma_{3}=-\frac{\partial \psi}{\partial x}, \\
\eta_{111}^{(1)}=\frac{2}{5}\left(\frac{\partial^{2} u}{\partial x^{2}}-z \frac{\partial^{2} \psi}{\partial x^{2}}+\frac{\partial w}{\partial x} \cdot \frac{\partial^{2} w}{\partial x^{2}}\right), \quad \eta_{333}^{(1)}=-\frac{1}{5}\left(\frac{\partial^{2} w}{\partial x^{2}}-2 \frac{\partial \psi}{\partial x}\right), \\
\eta_{113}^{(1)}=\eta_{311}^{(1)}=\eta_{131}^{(1)}=\frac{4}{15}\left(\frac{\partial^{2} w}{\partial x^{2}}-2 \frac{\partial \psi}{\partial x}\right), \quad \eta_{223}^{(1)}=\eta_{322}^{(1)}=\eta_{232}^{(1)}=-\frac{1}{15}\left(\frac{\partial^{2} w}{\partial x^{2}}-2 \frac{\partial \psi}{\partial x}\right), \\
\eta_{221}^{(1)}=\eta_{212}^{(1)}=\eta_{122}^{(1)}=\eta_{313}^{(1)}=\eta_{133}^{(1)}=\eta_{331}^{(1)}=-\frac{1}{5}\left(\frac{\partial^{2} u}{\partial x^{2}}-z \frac{\partial^{2} \psi}{\partial x^{2}}+\frac{\partial w}{\partial x} \cdot \frac{\partial^{2} w}{\partial x^{2}}\right) .
\end{gathered}
$$

The major components of the symmetric section of the stress tensor can be expressed by the kinematic parameters as follows:

$$
\begin{aligned}
\sigma_{11} & =(\lambda+2 \mu)\left(\frac{\partial u}{\partial x}-z \frac{\partial \psi}{\partial x}+\frac{1}{2}\left(\frac{\partial w}{\partial x}\right)^{2}\right), & \sigma_{22} & =\lambda\left(\frac{\partial u}{\partial x}-z \frac{\partial \psi}{\partial x}+\frac{1}{2}\left(\frac{\partial w}{\partial x}\right)^{2}\right), \\
\sigma_{33} & =\lambda\left(\frac{\partial u}{\partial x}-z \frac{\partial \psi}{\partial x}+\frac{1}{2}\left(\frac{\partial w}{\partial x}\right)^{2}\right), & \sigma_{13} & =\mu\left(\frac{\partial w}{\partial x}-\psi\right) .
\end{aligned}
$$

Note that $\sigma_{13}$ depends only on one direction. Due to taking the nonuniformity of the shear strain over the beam cross-section into account, a correction factor $k_{s}$, which depends on the shape of the beam section, is multiplied into the stress component $\sigma_{13}$ as follows [Hutchinson 2001; Wang et al. 2010]:

$$
\sigma_{13}=k_{s} \mu\left(\frac{\partial w}{\partial x}-\psi\right)
$$

At last, by inserting (6)-(9) into (3), the nonzero components of the higher-order stresses are obtained: 


$$
\begin{aligned}
& p_{1}=2 \mu l_{0}^{2}\left(\frac{\partial^{2} u}{\partial x^{2}}-z \frac{\partial^{2} \psi}{\partial x^{2}}+\frac{\partial w}{\partial x} \cdot \frac{\partial^{2} w}{\partial x^{2}}\right), \quad p_{3}=-2 \mu l_{0}^{2} \frac{\partial \psi}{\partial x}, \\
& \tau_{111}^{(1)}=\frac{4 \mu l_{1}^{2}}{5}\left(\frac{\partial^{2} u}{\partial x^{2}}-z \frac{\partial^{2} \psi}{\partial x^{2}}+\frac{\partial w}{\partial x} \cdot \frac{\partial^{2} w}{\partial x^{2}}\right), \quad \tau_{333}^{(1)}=-\frac{2 \mu l_{1}^{2}}{5}\left(\frac{\partial^{2} w}{\partial x^{2}}-2 \frac{\partial \psi}{\partial x}\right), \\
& \tau_{113}^{(1)}=\tau_{311}^{(1)}=\tau_{131}^{(1)}=\frac{8 \mu l_{1}^{2}}{15}\left(\frac{\partial^{2} w}{\partial x^{2}}-2 \frac{\partial \psi}{\partial x}\right), \quad \tau_{223}^{(1)}=\tau_{322}^{(1)}=\tau_{232}^{(1)}=-\frac{2 \mu l_{1}^{2}}{15}\left(\frac{\partial^{2} w}{\partial x^{2}}-2 \frac{\partial \psi}{\partial x}\right), \\
& \tau_{221}^{(1)}=\tau_{212}^{(1)}=\tau_{122}^{(1)}=\tau_{313}^{(1)}=\tau_{133}^{(1)}=\tau_{331}^{(1)}=-\frac{2 \mu l_{1}^{2}}{5}\left(\frac{\partial^{2} u}{\partial x^{2}}-z \frac{\partial^{2} \psi}{\partial x^{2}}+\frac{\partial w}{\partial x} \cdot \frac{\partial^{2} w}{\partial x^{2}}\right) \\
& m_{12}^{s}=m_{21}^{s}=-\frac{\mu l_{2}^{2}}{2}\left(\frac{\partial \psi}{\partial x}+\frac{\partial^{2} w}{\partial x^{2}}\right) .
\end{aligned}
$$

The initial axial stress in the beam, which is uniformly distributed in a section, can be written as $\sigma_{11}^{(0)}(x)=$ $N_{0}(x) / A$. Accordingly, (6) and (8) for $\varepsilon_{11}$ and $\sigma_{11}$ do not express the total values of the strain and the stress changes with respect to the fully unloaded conditions, but only express them with respect to the initial configuration.

In the following, the strain energy resulted from the advent of a variation in the classical and higherorder stresses with respect to the initial configuration denoted by $U_{1}$, the strain energy due to the presence of the initial axial stresses and the next imposed stretching denoted by $U_{2}$, and the beam's kinetic energy, $T$, are expressed as:

$$
\begin{aligned}
& U_{1}=\frac{1}{2} \int_{0}^{L} \int_{A}\left(\sigma_{i j} \varepsilon_{i j}+p_{i} \gamma_{i}+\tau_{i j k}^{(1)} \eta_{i j k}^{(1)}+m_{i j}^{s} \chi_{i j}^{s}\right) d A d x=\frac{1}{2} \int_{0}^{L} \int_{A}\left\{(\lambda+2 \mu)\left(\frac{\partial u}{\partial x}-z \frac{\partial \psi}{\partial x}+\frac{1}{2}\left(\frac{\partial w}{\partial x}\right)^{2}\right)^{2}\right. \\
& +k_{s} \mu\left(\frac{\partial w}{\partial x}-\psi\right)^{2}+2 \mu l_{0}^{2}\left(\frac{\partial \psi}{\partial x}\right)^{2}+\left(2 \mu l_{0}^{2}+\frac{20 \mu l_{1}^{2}}{25}\right)\left(\frac{\partial^{2} u}{\partial x^{2}}-z \frac{\partial^{2} \psi}{\partial x^{2}}+\frac{\partial w}{\partial x} \cdot \frac{\partial^{2} w}{\partial x^{2}}\right)^{2} \\
& \left.+\frac{40 \mu l_{1}^{2}}{75}\left(\frac{\partial^{2} w}{\partial x^{2}}-2 \frac{\partial \psi}{\partial x}\right)^{2}+\frac{\mu l_{2}^{2}}{4}\left(\frac{\partial \psi}{\partial x}+\frac{\partial^{2} w}{\partial x^{2}}\right)^{2}\right\} d A d x \\
& U_{2}=\int_{0}^{L} \frac{N_{0}}{A} \int_{A}\left(\frac{\partial u}{\partial x}-z \frac{\partial \psi}{\partial x}+\frac{1}{2}\left(\frac{\partial w}{\partial x}\right)^{2}\right) d A d x, \quad T=\frac{1}{2} \int_{0}^{L} \int_{A} \rho\left\{\left(\frac{\partial u}{\partial t}-z \frac{\partial \psi}{\partial t}\right)^{2}+\left(\frac{\partial w}{\partial t}\right)^{2}\right\} d A d x,
\end{aligned}
$$

where $\rho$ and $I$ are the density and area moments of inertia of sections with respect to the $y$-axis, respectively, and the latter is given by $I=\int_{A} z^{2} d A$. Also, note that $\int_{A} z d A=0$. Hence, for the total potential energy $U=U_{1}+U_{2}$ and the kinetic energy we will have

$$
\begin{array}{r}
U=\frac{1}{2} \int_{0}^{L}\left\{k_{1}\left(\frac{\partial u}{\partial x}+\frac{1}{2}\left(\frac{\partial w}{\partial x}\right)^{2}\right)^{2}+k_{2}\left(\frac{\partial \psi}{\partial x}\right)^{2}+k_{3}\left(\frac{\partial w}{\partial x}-\psi\right)^{2}+k_{4}\left(\frac{\partial^{2} \psi}{\partial x^{2}}\right)^{2}+k_{5}\left(\frac{\partial^{2} u}{\partial x^{2}}+\frac{\partial w}{\partial x} \cdot \frac{\partial^{2} w}{\partial x^{2}}\right)^{2}\right. \\
\left.+k_{6}\left(\frac{\partial^{2} w}{\partial x^{2}}-2 \frac{\partial \psi}{\partial x}\right)^{2}+k_{7}\left(\frac{\partial \psi}{\partial x}+\frac{\partial^{2} w}{\partial x^{2}}\right)^{2}+N_{0}\left(2 \frac{\partial u}{\partial x}+\left(\frac{\partial w}{\partial x}\right)^{2}\right)\right\} d x,
\end{array}
$$

$T=\frac{1}{2} \int_{0}^{L}\left\{\rho A\left(\frac{\partial u}{\partial t}\right)^{2}+\rho I\left(\frac{\partial \psi}{\partial t}\right)^{2}+\rho A\left(\frac{\partial w}{\partial t}\right)^{2}\right\} d x$ 
where

$$
\begin{aligned}
& k_{1}=(\lambda+2 \mu) A, \quad k_{2}=(\lambda+2 \mu) I+2 \mu A l_{0}^{2}, \quad k_{3}=k_{s} \mu A, \quad k_{4}=\mu I\left(2 l_{0}^{2}+\frac{4}{5} l_{1}^{2}\right), \\
& k_{5}=\mu A\left(2 l_{0}^{2}+\frac{4}{5} l_{1}^{2}\right), \quad k_{6}=\frac{8}{15} \mu A l_{1}^{2}, \quad k_{7}=\frac{1}{4} \mu A l_{2}^{2} .
\end{aligned}
$$

The external induced work associated with the body forces, body couples, and boundary surface tractions is

$$
\begin{aligned}
W^{\mathrm{ext}}=\int_{0}^{L}(G(x, t) u+ & \left.F(x, t) w+C(x, t) \theta_{2}\right) d x \\
& +\left.\left(\hat{N} u+\hat{V} w+\hat{M}_{\sigma} \psi+\hat{M}_{M} \frac{\partial w}{\partial x}+\hat{P}\left(\frac{\partial u}{\partial x}+\frac{1}{2}\left(\frac{\partial w}{\partial x}\right)^{2}\right)+\hat{Q} \frac{\partial \psi}{\partial x}\right)\right|_{x=0} ^{x=L},
\end{aligned}
$$

in which $\hat{N}$ is the axial resultant force of normal stresses $\sigma_{x x}+N_{0} / A, \hat{V}$ denotes the transverse resultant force of the shear stresses and $\hat{M}_{M}$ the resultant moment in a section caused by the classical and higherorder stress components, and $\hat{M}_{\sigma}$ is the resultant moment around the $y$-axis due to the couple stresses $m_{x y}$ at sections. In addition, the higher-order resultants in a section are denoted by $\hat{P}$ and $\hat{Q}$, which are due to higher-order stresses acting on that section.

By employing the Hamilton principle,

$$
\delta \int_{t_{1}}^{t_{2}}\left(T-U+W^{\mathrm{ext}}\right) d t=0
$$

taking the variation of $u, w$, and $\psi$, integrating by parts, and finally the coefficients of $\delta u, \delta w$, and $\delta \psi$ equal to zero, we obtain the governing equations of motion

$$
\begin{gathered}
\frac{\partial}{\partial x}\left\{N_{0}+k_{1}\left(\frac{\partial u}{\partial x}+\frac{1}{2}\left(\frac{\partial w}{\partial x}\right)^{2}\right)-k_{5} \frac{\partial^{2}}{\partial x^{2}}\left(\frac{\partial u}{\partial x}+\frac{1}{2}\left(\frac{\partial w}{\partial x}\right)^{2}\right)\right\}+G(x, t)=\rho A \frac{\partial^{2} u}{\partial t^{2}} \\
\frac{\partial}{\partial x}\left\{\left[N_{0}+k_{1}\left(\frac{\partial u}{\partial x}+\frac{1}{2}\left(\frac{\partial w}{\partial x}\right)^{2}\right)-k_{5} \frac{\partial^{2}}{\partial x^{2}}\left(\frac{\partial u}{\partial x}+\frac{1}{2}\left(\frac{\partial w}{\partial x}\right)^{2}\right)\right] \frac{\partial w}{\partial x}\right\}+k_{3}\left(\frac{\partial^{2} w}{\partial x^{2}}-\frac{\partial \psi}{\partial x}\right) \\
+\left(k_{6}-k_{7}\right) \frac{\partial^{4} w}{\partial x^{4}}-\left(2 k_{6}+k_{7}\right) \frac{\partial^{3} \psi}{\partial x^{3}}+F(x, t)+\frac{1}{2} \frac{\partial C(x, t)}{\partial x}=\rho A \frac{\partial^{2} w}{\partial t^{2}} \\
k_{2} \frac{\partial^{2} \psi}{\partial x^{2}}+k_{3}\left(\frac{\partial w}{\partial x}-\psi\right)-k_{4} \frac{\partial^{4} \psi}{\partial x^{4}}+\left(k_{7}-2 k_{6}\right) \frac{\partial^{3} w}{\partial x^{3}}+\left(k_{7}+4 k_{6}\right) \frac{\partial^{2} \psi}{\partial x^{2}}-\frac{C(x, t)}{2}=\rho I \frac{\partial^{2} \psi}{\partial t^{2}}
\end{gathered}
$$

and the boundary conditions:

$$
\begin{aligned}
& \left.\left(N_{0}+k_{1}\left(\frac{\partial u}{\partial x}+\frac{1}{2}\left(\frac{\partial w}{\partial x}\right)^{2}\right)-k_{5} \frac{\partial^{2}}{\partial x^{2}}\left(\frac{\partial u}{\partial x}+\frac{1}{2}\left(\frac{\partial w}{\partial x}\right)^{2}\right)-\hat{N}\right)\right|_{x=0, L}=0 \quad \text { or }\left.\quad \delta u\right|_{x=0, L}=0, \\
& \left.\left(k_{2} \frac{\partial \psi}{\partial x}-k_{4} \frac{\partial^{3} \psi}{\partial x^{3}}+\left(k_{7}-2 k_{6}\right) \frac{\partial^{2} w}{\partial x^{2}}+\left(k_{7}+4 k_{6}\right) \frac{\partial \psi}{\partial x}-\hat{M}_{\sigma}\right)\right|_{x=0, L}=0 \quad \text { or }\left.\quad \delta \psi\right|_{x=0, L}=0
\end{aligned}
$$




$$
\begin{aligned}
& \left(N_{0}+k_{1}\left(\frac{\partial u}{\partial x}+\frac{1}{2}\left(\frac{\partial w}{\partial x}\right)^{2}\right)-k_{5} \frac{\partial^{2}}{\partial x^{2}}\left(\frac{\partial u}{\partial x}+\frac{1}{2}\left(\frac{\partial w}{\partial x}\right)^{2}\right)\right) \frac{\partial w}{\partial x} \\
& +k_{3}\left(\frac{\partial w}{\partial x}-\psi\right)-\left(k_{6}+k_{7}\right) \frac{\partial^{3} w}{\partial x^{3}}+\left(2 k_{6}-k_{7}\right) \frac{\partial^{2} \psi}{\partial x^{2}}+\frac{C(x, t)}{2}-\left.\hat{V}\right|_{x=0, L}=0 \quad \text { or }\left.\quad \delta w\right|_{x=0, L}=0 \text {, } \\
& \left.\left(k_{5} \frac{\partial}{\partial x}\left(\frac{\partial u}{\partial x}+\frac{1}{2}\left(\frac{\partial w}{\partial x}\right)^{2}\right)-\hat{P}\right)\right|_{x=0, L}=0 \quad \text { or }\left.\quad \delta\left(\frac{\partial u}{\partial x}+\frac{1}{2}\left(\frac{\partial w}{\partial x}\right)^{2}\right)\right|_{x=0, L}=0, \\
& \left.\left(k_{4} \frac{\partial^{2} \psi}{\partial x^{2}}-\hat{Q}\right)\right|_{x=0, L}=0 \quad \text { or } \\
& \left.\left(\left(k_{6}+k_{7}\right) \frac{\partial^{2} w}{\partial x^{2}}+\left(k_{7}-2 k_{6}\right) \frac{\partial \psi}{\partial x}-\hat{M}_{M}\right)\right|_{x=0, L}=0 \quad \text { or }\left.\quad \delta\left(\frac{\partial w}{\partial x}\right)\right|_{x=0, L}=0 .
\end{aligned}
$$

Consequently, on the basis of the strain gradient theory the governing equations of motion of a nonlinear size-dependent Timoshenko beam are achieved. Neglecting the nonlinear terms, body forces, body couples, and boundary surface tractions in (16b) and (16c), these equations can be reduced to those given by [Wang et al. 2010]. In the cases associated with a thin beam with a huge aspect ratio, same as in the classical beam theories, Poisson's effect is no more effective and can be negligible, that is, $v=0$; but in other cases it must be considered to get reliable outcomes. Letting the material length-scale parameters $l_{0}, l_{1}$, and $l_{2}$ equal zero, the governing equations and corresponding boundary conditions related to a nonlinear Timoshenko beam modeled by classical beam theory will be achieved. Moreover, the governing equations and boundary conditions of a nonlinear Timoshenko beam modeled via the modified couple stress theory can be achieved if only $l_{0}=l_{1}=0$ and $l_{2}=l$ (see [Asghari et al. 2010b]). Additionally, by neglecting the shear deformation, governing equations and boundary conditions corresponding to the microscale Euler-Bernoulli beam model based on the strain gradient elasticity theory will be attained (see [Kahrobaiyan et al. 2011]).

\section{Governing equations of motion of a simply supported beam with immovable ends and corresponding boundary conditions: A specific case}

Herein, specifically simply supported beams with immobile ends are considered and the strain gradient formulation of the earlier section is developed for this case. The relevant boundary conditions are

$$
u(0, t)=u(L, t)=0 \quad \text { and } \quad w(0, t)=w(L, t)=0 .
$$

We assume $\hat{P}, \hat{Q}, \hat{M}_{\sigma}$, and $\hat{M}_{M}$ are zero at the end sections. Accordingly, the other boundary conditions can be obtained as follows:

$$
\begin{aligned}
& \left.\frac{\partial}{\partial x}\left(\frac{\partial u}{\partial x}+\frac{1}{2}\left(\frac{\partial w}{\partial x}\right)^{2}\right)\right|_{x=0, L}=0, \\
& \left.\left(k_{2} \frac{\partial \psi}{\partial x}-k_{4} \frac{\partial^{3} \psi}{\partial x^{3}}+\left(k_{7}-2 k_{6}\right) \frac{\partial^{2} w}{\partial x^{2}}+\left(k_{7}+4 k_{6}\right) \frac{\partial \psi}{\partial x}\right)\right|_{x=0, L}=0, \\
& \left.\left(\left(k_{6}+k_{7}\right) \frac{\partial^{2} w}{\partial x^{2}}+\left(k_{7}-2 k_{6}\right) \frac{\partial \psi}{\partial x}\right)\right|_{x=0, L}=0,\left.\quad\left(\frac{\partial \psi}{\partial x}\right)\right|_{x=0, L}=0 .
\end{aligned}
$$


Compared to the inertia term in the transverse direction, the horizontal inertia force can be neglected [Asghari et al. 2010b]. Considering this assumption and assuming $N_{0}$ independent of $x$, in the absence of axial body forces (that is, $G(x, t)=0$ ), the equation of motion in the axial direction (16a) and the boundary condition (18a) give

$$
k_{1}\left(\frac{\partial u}{\partial x}+\frac{1}{2}\left(\frac{\partial w}{\partial x}\right)^{2}\right)+N_{0}=N(t)
$$

In the case of axially immobile ends $(u(0, t)=u(L, t)=0)$, integrating (19) between the limits 0 and $L$ leads to

$$
N(t)=N_{0}+\tilde{N}(t)=N_{0}+\frac{k_{1}}{2 L} \int_{0}^{L}\left(\frac{\partial w}{\partial x}\right)^{2} d x
$$

The axial force variation in the beam with respect to the initial configuration is denoted by $\tilde{N}(t)$ and is indeed due to the extension resulting from the transverse deformation. Inserting (20) into (16b), the equations of motion can be rewritten in the following form:

$$
N \frac{\partial^{2} w}{\partial x^{2}}+k_{3}\left(\frac{\partial^{2} w}{\partial x^{2}}-\frac{\partial \psi}{\partial x}\right)+\left(k_{6}-k_{7}\right) \frac{\partial^{4} w}{\partial x^{4}}-\left(2 k_{6}+k_{7}\right) \frac{\partial^{3} \psi}{\partial x^{3}}+F(x, t)+\frac{1}{2} \frac{\partial C(x, t)}{\partial x}=\rho A \frac{\partial^{2} w}{\partial t^{2}} .
$$

The governing equations of the dynamic behavior of a nonlinear size-dependent Timoshenko beam with two immobile ends modeled on the basis of strain gradient theory are represented in (16c) and (21) and the associated boundary conditions are given by $w(0, t)=w(L, t)=0$ and by (18b)-(18c). If the material length-scale parameters $l_{0}, l_{1}$, and $l_{2}$ are set to be zero, the governing equations and corresponding boundary conditions of a nonlinear Timoshenko beam with immobile supports modeled by classical beam theory will be attained (see [Ansari and Ramezannezhad 2011]). Furthermore, to achieve the governing equations and boundary conditions of a nonlinear Timoshenko beam with two immobile supports modeled by modified couple stress theory, it is enough to let $l_{0}=l_{1}=0$ and $l_{2}=l$.

\section{Investigation of the free vibration of a simply supported beam}

Herein, as an example, based on strain gradient theory, the free vibration of nonlinear size-dependent Timoshenko beams with simply supported boundary conditions and immovable ends under the assumption $N_{0}=F=C=0$ are investigated.

For beams with simply supported edges, the solutions of the governing equations can be introduced as $\psi(x, t)=\Psi(t) \cos (m \pi x / L)$ and $w(x, t)=W(t) \sin (m \pi x / L)$. Inserting these solutions into (16c) and (21) results in

$$
\begin{aligned}
\ddot{W}+\frac{\pi^{4} m^{4}(\lambda+2 \mu)}{4 \rho L^{4}} W^{3}+\left(\frac{k_{s} \mu \pi^{2} m^{2}}{\rho L^{2}}-\frac{8 \mu l_{1}^{2} \pi^{4} m^{4}}{15 \rho L^{4}}+\frac{\mu l_{2}^{2} \pi^{4} m^{4}}{4 \rho L^{4}}\right) W \\
+\left(\frac{16 \mu l_{1}^{2} \pi^{3} m^{3}}{15 \rho L^{3}}-\frac{k_{s} \mu m \pi}{\rho L}+\frac{\mu l_{2}^{2} \pi^{3} m^{3}}{\rho L^{3}}\right) \Psi=0,
\end{aligned}
$$




$$
\begin{array}{r}
\ddot{\Psi}+\left(\frac{\pi^{2} m^{2}(\lambda+2 \mu)}{\rho L^{2}}+\frac{2 \mu A \pi^{2} m^{2} l_{0}^{2}}{\rho I L^{2}}+\frac{k_{s} \mu A}{\rho I}+\frac{2 \mu l_{0}^{2} \pi^{4} m^{4}}{\rho L^{4}}+\frac{4 \mu l_{1}^{2} \pi^{4} m^{4}}{5 \rho L^{4}}+\frac{32 \mu A l_{1}^{2} \pi^{2} m^{2}}{15 \rho I L^{2}}+\frac{\mu A l_{2}^{2} \pi^{2} m^{2}}{4 \rho I L^{2}}\right) \Psi \\
+\left(\frac{\mu A l_{2}^{2} \pi^{3} m^{3}}{4 \rho I L^{3}}-\frac{k_{s} \mu A m \pi}{\rho I L}-\frac{16 \mu A l_{1}^{2} \pi^{3} m^{3}}{15 \rho I L^{3}}\right) W=0 . \quad(22 \mathrm{~b})
\end{array}
$$

The governing equations must be expressed in nondimensional form by using the following nondimensional parameters:

$$
\begin{aligned}
& \omega_{l}=\sqrt{\frac{\pi^{2}(\lambda+2 \mu)}{\rho L^{2}}}, \quad \omega_{s}=\sqrt{\frac{k_{s} \mu A}{\rho I}}, \quad \omega_{r}=\sqrt{\frac{\pi^{2} k_{s} \mu}{\rho L^{2}}}, \\
& \omega_{m}=\sqrt{\frac{\mu \pi^{2}}{\rho L^{2}}}, \quad \omega_{n}=\sqrt{\frac{\mu A}{\rho I}}, \quad\left(\alpha_{0}, \alpha_{1}, \alpha_{2}\right)=\frac{\pi^{2}}{4 L^{2}}\left(l_{0}^{2}, l_{1}^{2}, l_{2}^{2}\right), \quad \tau=\omega t .
\end{aligned}
$$

In order to achieve an accurate analytical solution, the nonlinear equations resulting from (22) are solved utilizing the harmonic balance method. According to this method, the solutions are assumed as a truncated Fourier series of the form [Gao and Lei 2009]

$$
W(\tau)=\Gamma_{1} \cos \tau+\Gamma_{1} \cos 3 \tau, \quad \Psi(\tau)=\Lambda_{1} \cos \tau+\Lambda_{2} \cos 3 \tau .
$$

Inserting the given solution into (22), substituting the above Fourier series, ignoring the higher-order harmonics than ones comprised in the original assumed solutions and setting the coefficients of like cosine harmonics (such as the $\cos \tau$ and $\cos 3 \tau$ terms) equal to zero, a set of algebraic equations will be achieved as follows:

$$
\begin{array}{r}
-\omega^{2} \frac{\Gamma_{1}}{L}+\left(m^{4} \alpha_{2} \omega_{m}^{2}+m^{2} \pi \omega_{r}^{2}-\frac{32 m^{4}}{15} \alpha_{1} \omega_{m}^{2}\right) \frac{\Gamma_{1}}{L}+\left(\frac{4 m^{3} \alpha_{2}}{\pi} \omega_{m}^{2}+\frac{64 \alpha_{1} m^{3}}{15 \pi} \omega_{m}^{2}-\frac{m \omega_{r}^{2}}{\pi}\right) \Lambda_{1} \\
+\frac{\pi^{2} m^{4} \omega_{l}^{2}}{4 L^{3}}\left(\frac{3}{4} \Gamma_{1}^{3}+\frac{3}{4} \Gamma_{1}^{2} \Gamma_{2}+\frac{3}{2} \Gamma_{2}^{2} \Gamma_{1}\right)=0 \\
-\omega^{2} \Lambda_{1}+\left(m^{2} \omega_{l}^{2}+8 m^{2} \alpha_{0} \omega_{n}^{2}+\omega_{s}^{2}+8 m^{4} \alpha_{0} \omega_{m}^{2}+\frac{16}{5} m^{4} \alpha_{1} \omega_{m}^{2}+\frac{128 m^{2}}{15} \alpha_{1} \omega_{n}^{2}+m^{2} \alpha_{2} \omega_{n}^{2}\right) \Lambda_{1} \\
+\left(\pi m^{3} \alpha_{2} \omega_{n}^{2}-m \pi \omega_{s}^{2}-\frac{64 \pi m^{3}}{15} \alpha_{1} \omega_{n}^{2}\right) \frac{\Gamma_{1}}{L}=0 \\
-9 \omega^{2} \frac{\Gamma_{2}}{L}+\left(m^{4} \alpha_{2} \omega_{m}^{2}+m^{2} \pi \omega_{r}^{2}-\frac{32 m^{4}}{15} \alpha_{1} \omega_{m}^{2}\right) \frac{\Gamma_{2}}{L}+\left(\frac{4 m^{3} \alpha_{2}}{\pi} \omega_{m}^{2}+\frac{64 \alpha_{1} m^{3}}{15 \pi} \omega_{m}^{2}-\frac{m \omega_{r}^{2}}{\pi}\right) \Lambda_{2} \\
+\frac{\pi^{2} m^{4} \omega_{l}^{2}}{4 L^{3}}\left(\frac{1}{4} \Gamma_{1}^{3}+\frac{3}{2} \Gamma_{1}^{2} \Gamma_{2}+\frac{3}{4} \Gamma_{2}^{3}\right)=0 \\
-9 \omega^{2} \Lambda_{1}+\left(m^{2} \omega_{l}^{2}+8 m^{2} \alpha_{0} \omega_{n}^{2}+\omega_{s}^{2}+8 m^{4} \alpha_{0} \omega_{m}^{2}+\frac{16}{5} m^{4} \alpha_{1} \omega_{m}^{2}+\frac{128 m^{2}}{15} \alpha_{1} \omega_{n}^{2}+m^{2} \alpha_{2} \omega_{n}^{2}\right) \Lambda_{2} \\
+\left(\pi m^{3} \alpha_{2} \omega_{n}^{2}-m \pi \omega_{s}^{2}-\frac{64 \pi m^{3}}{15} \alpha_{1} \omega_{n}^{2}\right) \frac{\Gamma_{2}}{L}=0
\end{array}
$$

Utilizing the Newton-Raphson method, these nonlinear equations can be solved for $\omega, \Lambda_{1}, \Lambda_{2}$, and $\Gamma_{2}$ by choosing $\Gamma_{1}$ as a determined parameter. 
A microbeam made of epoxy with mechanical properties $E=1.44 \mathrm{GPa}$ and $\rho=1.22 \times 10^{3} \mathrm{~kg} / \mathrm{m}^{3}$ [Maneschy et al. 1986] is considered under the assumption $l_{0}=l_{1}=l_{2}=l=17.6 \mu \mathrm{m}$ [Ma et al. 2008; Kong et al. 2009]. The shear coefficient $k_{s}$ is given as $(5+5 v) /(6+5 v)$ which is assessed as the most efficient description for a rectangular cross-section beam [Ke et al. 2012].

As was mentioned, if the material length-scale parameters are set as $l_{0}=l_{1}=0$ and $l_{2}=l$ and the nonlinearity is neglected, the linear free vibration of the homogeneous microbeams presented in [Ma et al. 2008 ] can be degenerated. To check the validity and accuracy of the present analysis, the first four natural frequencies of a simply supported Timoshenko microbeam with $E=1.44 \mathrm{GPa}, \rho=1.22 \times 10^{3} \mathrm{~kg} / \mathrm{m}^{3}$, $L / h=10$, and $l=17.6 \mu \mathrm{m}$ obtained from the present analysis are compared with those of Ma et al., given in Table 1. As can be seen from the table, a good agreement has been achieved. The small difference between our results and those of Ma et al. is due to considering the Poisson effect. We observe that these differences become really significant at lower $l / h$ and higher modes.

Table 2 compares the results obtained with the present method, based on classical, modified couple stress, and strain gradient theories, with those reported in [Ansari et al. 2011] for the same theories. As in that paper, we report the dimensionless natural frequencies, $\Omega=\omega L \sqrt{\rho_{m} /\left(\lambda_{m}+2 \mu_{m}\right)}$, of simply supported homogeneous metal (Al) and ceramic ( $\mathrm{SiC})$ microbeams with material properties $E_{m}=70 \mathrm{GPa}$, $v_{m}=0.3$, and $\rho_{m}=2702 \mathrm{~kg} / \mathrm{m}^{3}$ for $\mathrm{Al}$ and $E_{c}=427 \mathrm{GPa}, v_{c}=0.17$, and $\rho_{c}=3100 \mathrm{~kg} / \mathrm{m}^{3}$ for SiC. The other parameters used are $L / h=10, h / l=2$, and $l=15 \mu \mathrm{m}$. Excellent agreement can be observed between the present results and the earlier ones.

Table 3 gives the nonlinear frequency ratios for simply supported homogeneous microbeams with the material properties $E=70 \mathrm{GPa}, \rho=2702 \mathrm{~kg} / \mathrm{m}^{3}$, and $v=0.3$ [Ke et al. 2012] based on modified couple stress theory. The results of Ke et al. are also provided for comparison. The other parameters used in the example are $L / h=12, h / l=2$, and $l=15 \mu \mathrm{m}$ [Ke et al. 2012]. Excellent agreement can be observed

\begin{tabular}{|ccccccc|}
\hline Mode & \multicolumn{2}{c}{$h / l=1$} & \multicolumn{2}{c}{$h / l=3$} & \multicolumn{2}{c|}{$h / l=10$} \\
& present & Ma et al. & present & Ma et al. & present & Ma et al. \\
1 & 0.6733 & 0.6723 & 0.1393 & 0.1391 & 0.0377 & 0.0377 \\
2 & 2.4643 & 2.4530 & 0.5182 & 0.5163 & 0.1402 & 0.1396 \\
3 & 4.9741 & 4.9368 & 1.0588 & 1.0519 & 0.2858 & 0.2838 \\
4 & 7.9372 & 7.8606 & 1.6966 & 1.6814 & 0.4557 & 0.4514 \\
\hline
\end{tabular}

Table 1. Comparison of the first four linear natural frequencies (in MHz) of microbeams with consideration of size effect. Literature results are from [Ma et al. 2008].

\begin{tabular}{|ccccc|}
\hline Theory & \multicolumn{2}{c}{ Ceramic } & \multicolumn{2}{c|}{ Metal } \\
& present & previous & present & previous \\
Classical & 0.5778 & 0.5776 & 0.2810 & 0.2854 \\
Modified couple stress & 0.8541 & 0.8538 & 0.3820 & 0.3863 \\
Strain gradient & 1.2705 & 1.2608 & 0.5471 & 0.5430 \\
\hline
\end{tabular}

Table 2. Comparison of the dimensionless natural frequencies of microbeams corresponding to different beam models. "Previous" refers to results from [Ansari et al. 2011]. 


\begin{tabular}{|ccccc|}
\hline & $\Omega_{\text {Linear }}=\omega L \sqrt{\rho / E}$ & \multicolumn{3}{c|}{ Nonlinear frequency ratio } \\
& & $w_{\max } / h=0.2$ & $w_{\max } / h=0.4$ & $w_{\max } / h=0.6$ \\
present & 0.3187 & 1.0338 & 1.1259 & 1.2654 \\
[Ke et al. 2012] & 0.3186 & 1.0340 & 1.1265 & 1.2671 \\
\hline
\end{tabular}

Table 3. Comparison of the dimensionless natural frequency and nonlinear frequency ratios of microbeams.

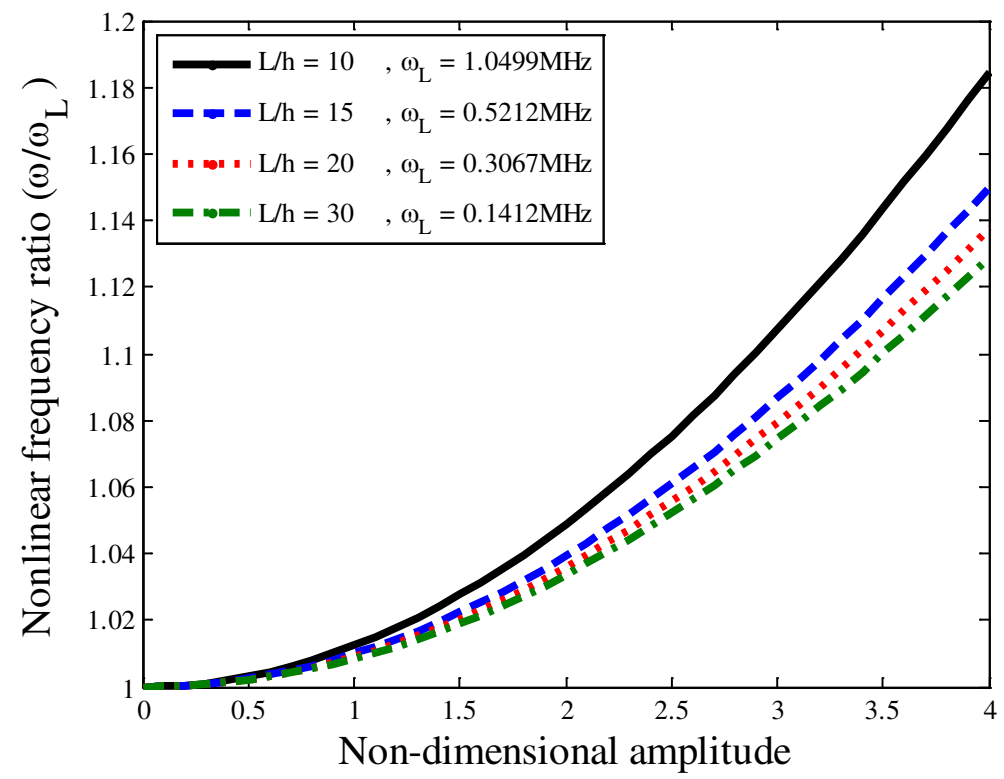

Figure 2. Effect of the length-to-thickness ratio $(L / h)$ on the nonlinear frequency ratio, for $h=l$ and $b=2 h$.

between our results and those of Ke et al.

The nonlinear free vibration of a simply supported beam is investigated in Figures 2-6. The linear fundamental frequencies $\left(\omega_{L}\right)$ are also given in Figures 2-5. Figure 2 illustrates the nonlinear frequency ratio versus the vibration amplitude for different length-to-thickness ratios for the geometric parameters $h=l, b=2 h$. It is seen that the beam exhibits a typical hard-spring behavior, that is, the nonlinear frequency ratio increases as the vibration amplitude increases. The length-to-thickness ratio has a significant effect on the nonlinear vibration behavior. At a given vibration amplitude, both the linear frequency and nonlinear frequency ratios decrease with the increase of the length-to-thickness ratio.

The effect of the ratio of the beam thickness to the material length-scale parameter $(h / l)$ on the nonlinear frequency ratio is investigated in Figure 3. This figure is illustrated for the geometric parameters $b=2 h$ and $L=20 h$ and reveals that as the ratio $h / l$ increases, unlike for the linear frequency, the nonlinear frequency ratio gets bigger. At higher amplitudes, the discrepancy between the curves becomes more prominent.

Figure 4 depicts the Poisson's ratio's influence on the linear and nonlinear frequencies for two different geometric parameters. For the case of $h=l, b=2 h$, and $L=30 h$, an increase in the Poisson's ratio 


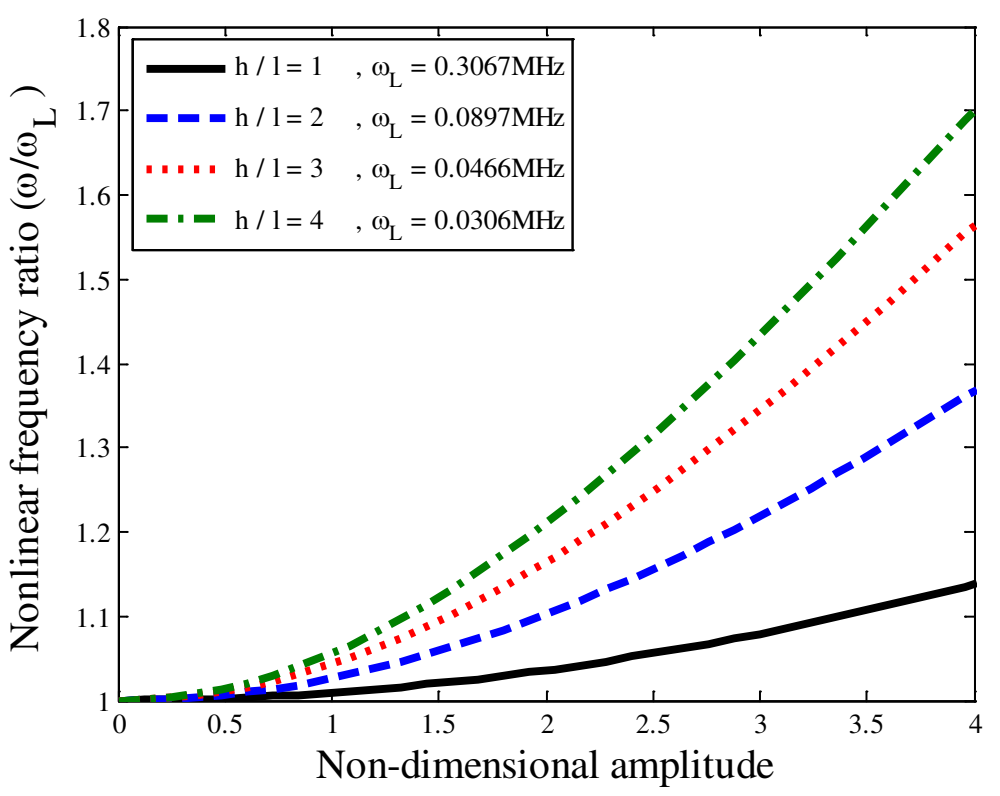

Figure 3. Effect of the ratio of the beam thickness to the material length scale parameter $(h / l)$ on the nonlinear frequency ratio, for $b=2 h$ and $L=20 h$.

leads to lower linear frequencies while the nonlinear frequency ratio becomes higher. In addition, for the geometric parameters $h=3 l, b=2 h$, and $L=20 h$, an increase in the Poisson's ratio results in higher nonlinear frequency ratios but results in unpredictable behavior of the linear frequency. This indicates that the size effect plays a key role in the investigation of the effect of Poisson's ratio on the natural frequency of microbeams.

Figure 5 illustrates the influences of additional material parameters on the nonlinear frequency ratio. The figure is plotted for the nonlinear frequency ratio versus the nondimensional amplitude for $h=2 l$, $b=2 h, L=10 h$, and $v=0.38$. As the figure indicates, the value of the nonlinear frequency ratio predicted by classical beam theory is more than that of the nonclassical beam theories. Also, according to the figure, strain gradient beam theory predicts the lowest nonlinear frequency ratio. In other words, the modified couple stress and classical beam theories tend to overestimate the nonlinear frequency ratio, especially when the amplitude gets higher. Furthermore, as the amplitude rises, the deviation between the theories becomes more prominent.

Another comparison between the classical and nonclassical beam theories is illustrated in Figure 6, but this time the figure shows the natural frequency of a microbeam assessed by the nonclassical and classical theories for two different Poisson's ratios. The trends are illustrated for a nondimensional vibrational amplitude $\left(w_{\max } / \sqrt{I / A}\right)$ equal to 3 . This figure indicates that the natural frequencies obtained by the nonclassical Timoshenko beam models are higher than the ones predicted by the classical model for both Poisson's ratios. The natural frequencies predicted by the strain gradient model are higher than those from the modified couple stress model. In other words, apart from strain gradient theory, the theories mentioned tend to underestimate the natural frequency. In addition, as can be deduced from this figure, for the classical beam model, the natural frequency with $v=0$ is always lower than that with $v=0.38$. 


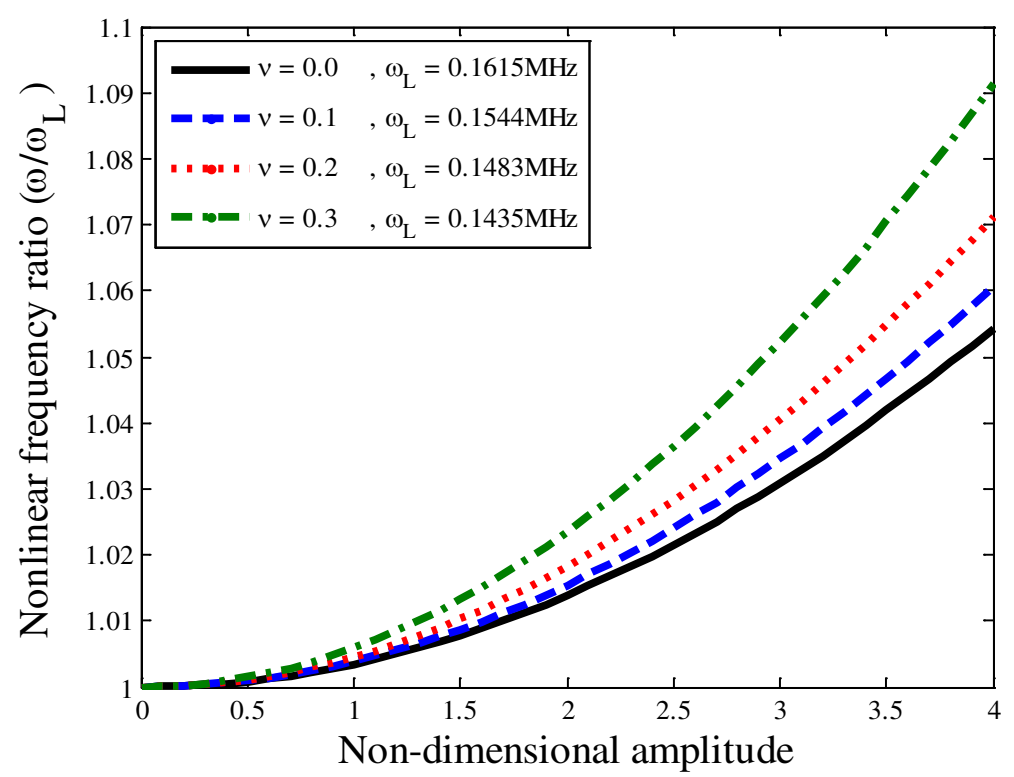

(a): $h=l, b=2 h, L=30 h$

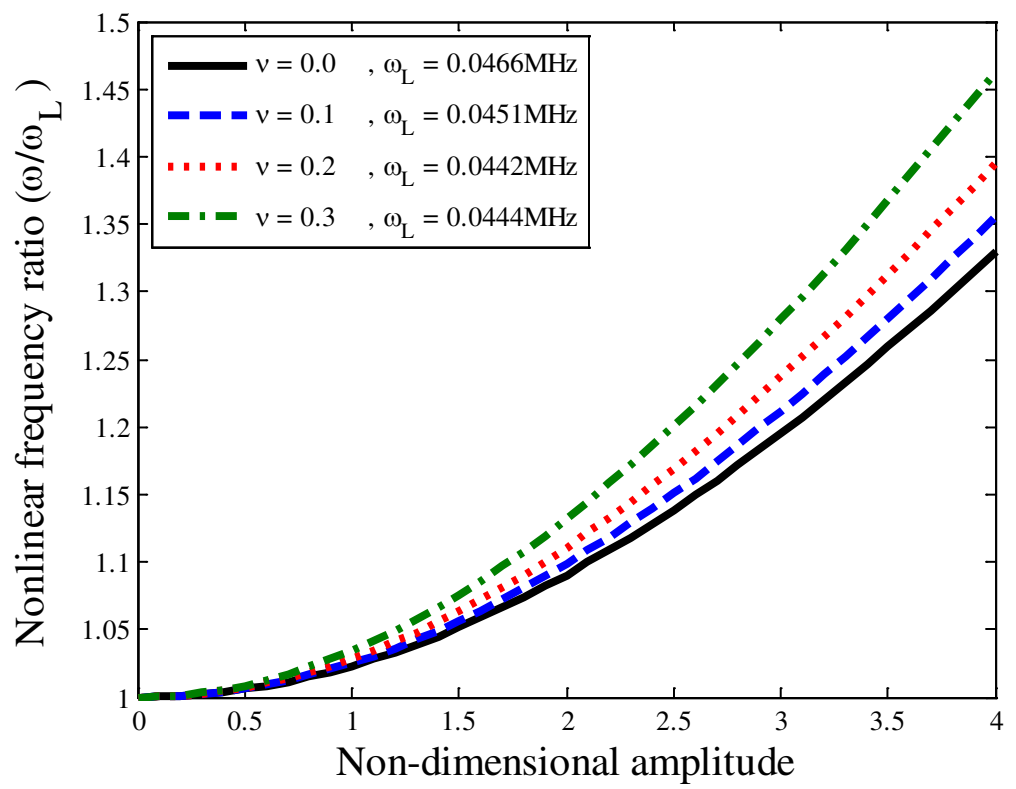

(b): $h=3 l, b=2 h, L=20 h$

Figure 4. Influence of the Poisson's ratio on the nonlinear frequency ratio for the given values of $h, b$, and $L$.

However, for the nonclassical beam theories, this is not true at low $h / l$. At low and high $h / l$, with an increase of the Poisson's ratio, the microbeam natural frequency corresponding to the nonclassical beam models decreases and increases, respectively. According to this figure, it can be concluded that the Poisson effect is more prominent at low thickness ratios. Hence, the reliability of the beam theories 


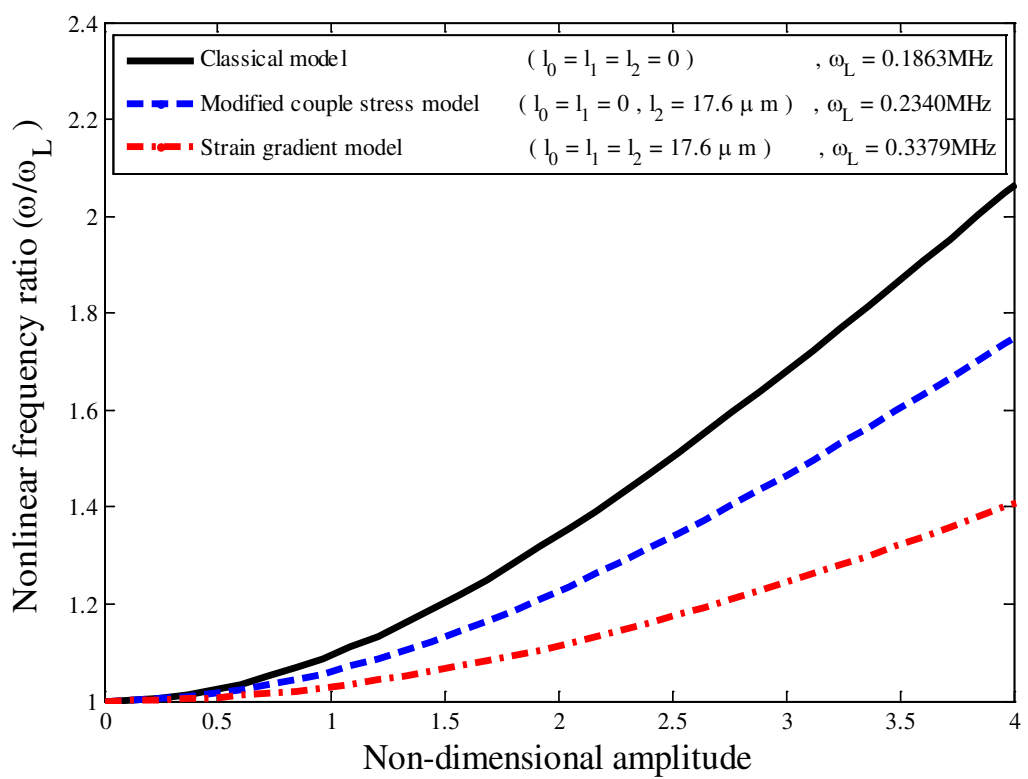

Figure 5. Comparison between classical and nonclassical beam theories and the influence of additional material parameters on the nonlinear frequency ratio, for $h=2 l$, $b=2 h, L=10 h$, and $v=0.38$.

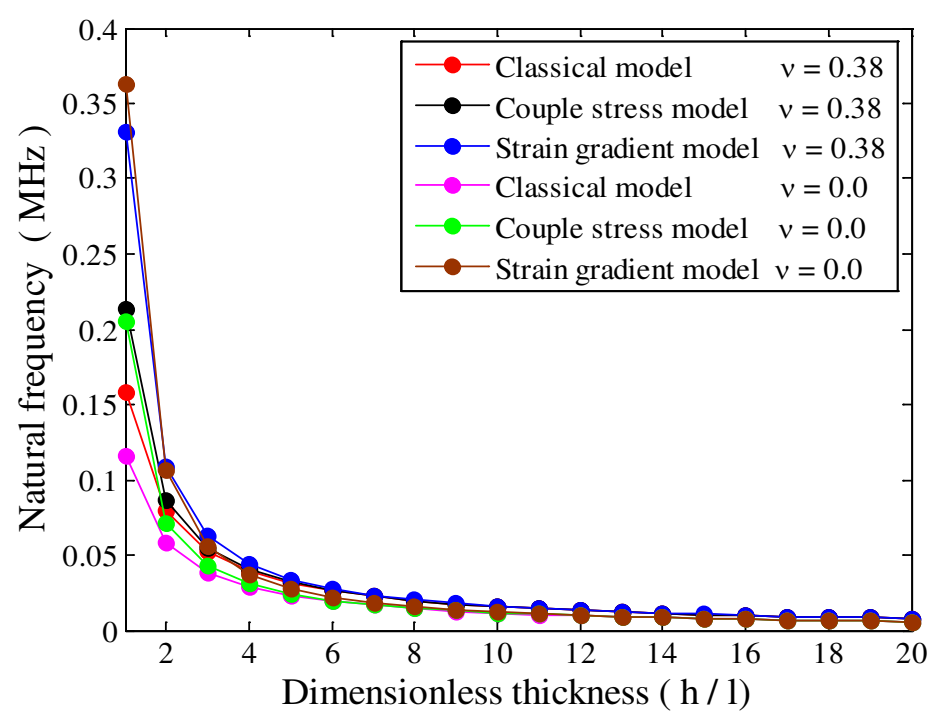

Figure 6. Nonlinear frequency versus the dimensionless thickness corresponding to two different values of Poisson's ratio and the classical and nonclassical beam theories, for $b=2 h$ and $L=20 h$.

which neglect the Poisson effect is questionable, so in order to obtain more accurate results, this effect must be incorporated. 


\section{Conclusion}

Based on strain gradient theory and using Hamilton's principle, a comprehensive geometrically nonlinear size-dependent Timoshenko beam model incorporating the Poisson effect was developed. The model contains the nonlinear governing partial differential equations of motions and the corresponding classical and nonclassical boundary conditions. The current nonclassical Timoshenko beam model encompasses the available modified couple stress and classical models and recovers the nonclassical Euler-Bernoulli beam model when $\psi=\partial w / \partial x$. As a specific case, the governing equations and the corresponding boundary conditions were solved by the use of the harmonic balance method to describe the large-amplitude sizedependent vibration behavior of simply supported microbeams. The numerical results obtained from the present model were compared with previously published results, from the linear strain gradient and the nonlinear and linear modified couple stress theories, as well as linear and nonlinear classical models, showing good agreement.

\section{References}

[Aifantis 1999] E. C. Aifantis, "Strain gradient interpretation of size effects", Int. J. Fract. 95 (1999), 299-314.

[Ansari and Ramezannezhad 2011] R. Ansari and H. Ramezannezhad, "Nonlocal Timoshenko beam model for the largeamplitude vibrations of embedded multiwalled carbon nanotubes including thermal effects", Physica E 43 (2011), 1171-1178.

[Ansari et al. 2011] R. Ansari, R. Gholami, and S. Sahmani, "Free vibration analysis of size-dependent functionally graded microbeams based on the strain gradient Timoshenko beam theory", Compos. Struct. 94 (2011), 221-228.

[Asghari et al. 2010a] M. Asghari, M. T. Ahmadian, M. H. Kahrobaiyan, and M. Rahaeifard, "On the size-dependent behavior of functionally graded micro-beams", Mater. Des. 31 (2010), 2324-2329.

[Asghari et al. 2010b] M. Asghari, M. H. Kahrobaiyan, and M. T. Ahmadian, "A nonlinear Timoshenko beam formulation based on the modified couple stress theory", Int. J. Eng. Sci. 48 (2010), 1749-1761.

[Coutu et al. 2004] R. A. Coutu, P. E. Kladitis, L. A. Starman, and J. R. Reid, "A comparison of micro-switch analytic, finite element, and experimental results", Sens. Actuators, A 115 (2004), 252-258.

[Craighead 2000] H. G. Craighead, "Nanoelectromechanical systems", Science 290 (2000), 1532-1535.

[Eringen 1972] A. C. Eringen, "Nonlocal polar elastic continua”, Int. J. Eng. Sci. 10 (1972), 1-16.

[Fleck and Hutchinson 1993] N. A. Fleck and J. W. Hutchinson, "Phenomenological theory for strain gradient effects in plasticity", J. Mech. Phys. Solids 41 (1993), 1825-1857.

[Fleck and Hutchinson 1997] N. A. Fleck and J. W. Hutchinson, "Strain gradient plasticity", Adv. Appl. Mech. 33 (1997), 295-361.

[Fleck and Hutchinson 2001] N. A. Fleck and J. W. Hutchinson, "A reformulation of strain gradient plasticity", J. Mech. Phys. Solids 49 (2001), 2245-2271.

[Fleck et al. 1994] N. A. Fleck, G. M. Muller, M. F. Ashby, and J. W. Hutchinson, "Strain gradient plasticity: theory and experiment", Acta Metall. Mater. 42 (1994), 475-487.

[Gao and Lei 2009] Y. Gao and F. Lei, "Small scale effects on the mechanical behaviors of protein microtubules based on the nonlocal elasticity theory", Biochem. Biophys. Res. Commun. 387 (2009), 467-471.

[Giannakopoulos and Stamoulis 2007] A. E. Giannakopoulos and K. Stamoulis, "Structural analysis of gradient elastic components", Int. J. Solids Struct. 44 (2007), 3440-3451.

[Gurtin et al. 1998] M. E. Gurtin, J. Weissmuller, and F. Larche, "The general theory of curved deformable interfaces in solids at equilibrium", Philos. Mag. A 78 (1998), 1093-1109.

[Hutchinson 2001] J. R. Hutchinson, "Shear coefficients for Timoshenko beam theory", J. Appl. Mech. 68 (2001), 87-92.

[Kahrobaiyan et al. 2011] M. H. Kahrobaiyan, M. Asghari, M. Rahaeifard, and M. T. Ahmadian, "A nonlinear strain gradient beam formulation", Int. J. Eng. Sci. 49:11 (2011), 1256-1267. 
[Kaneko 1975] T. Kaneko, “On Timoshenko's correction for shear in vibrating beams", J. Phys. D: Appl. Phys. 8 (1975), 1927-1936.

[Ke et al. 2012] L. L. Ke, Y. S. Wang, J. Yang, and S. Kitipornchai, "Nonlinear free vibration of size-dependent functionally graded microbeams", Int. J. Eng. Sci. 50:1 (2012), 256-267.

[Kong et al. 2008] S. Kong, S. Zhou, Z. Nie, and K. Wang, "The size-dependent natural frequency of Bernoulli-Euler microbeams", Int. J. Eng. Sci. 46 (2008), 427-437.

[Kong et al. 2009] S. Kong, S. Zhou, Z. Nie, and K. Wang, "Static and dynamic analysis of micro beams based on strain gradient elasticity theory”, Int. J. Eng. Sci. 47 (2009), 487-498.

[Lam et al. 2003] D. C. C. Lam, F. Yang, A. C. M. Chong, J. Wang, and P. Tong, "Experiments and theory in strain gradient elasticity”, J. Mech. Phys. Solids 51 (2003), 1477-1508.

[Lazopoulos 2004] K. A. Lazopoulos, "On the gradient strain elasticity theory of plates", Eur. J. Mech. A-Solid 23 (2004), 843-852.

[Lazopoulos and Lazopoulos 2010] K. A. Lazopoulos and A. K. Lazopoulos, "Bending and buckling of thin strain gradient elastic beams", Eur. J. Mech. A-Solid 29 (2010), 837-843.

[Lun et al. 2006] F. Y. Lun, P. Zhang, F. B. Gao, and H. G. Jia, "Design and fabrication of micro-optomechanical vibration sensor", Microfabrication Tech. 120 (2006), 61-64.

[Ma et al. 2008] H. M. Ma, X.-L. Gao, and J. N. Reddy, "A microstructure-dependent Timoshenko beam model based on a modified couple stress theory”, J. Mech. Phys. Solids 56 (2008), 3379-3391.

[Ma et al. 2010] H. M. Ma, X. L. Gao, and J. N. Reddy, "A non-classical Reddy-Levinson beam model based on a modified couple stress theory", Int. J. Multiscale Comput. Eng. 8 (2010), 167-180.

[Ma et al. 2011] H. M. Ma, X. L. Gao, and J. N. Reddy, "A non-classical Mindlin plate model based on a modified couple stress theory", Acta Mech. 220 (2011), 217-235.

[Mahdavi et al. 2008] M. H. Mahdavi, A. Farshidianfar, M. Tahani, S. Mahdavi, and H. Dalir, "A more comprehensive modeling of atomic force microscope cantilever", J. Ultramicroscopy 109 (2008), 54-60.

[Maneschy et al. 1986] C. E. Maneschy, Y. Miyano, M. Shimbo, and T. C. Woo, "Residual-stress analysis of an epoxy plate subjected to rapid cooling on both surfaces", Exp. Mech. 26 (1986), 306-312.

[Mindlin 1965] R. D. Mindlin, "Second gradient of strain and surface tension in linear elasticity", Int. J. Solids Struct. 1 (1965), 417-438.

[Mindlin and Tiersten 1962] R. D. Mindlin and H. F. Tiersten, "Effects of couple-stresses in linear elasticity", Arch. Ration. Mech. Anal. 11 (1962), 415-448.

[Moghimi Zand and Ahmadian 2009] M. Moghimi Zand and M. T. Ahmadian, "Vibrational analysis of electrostatically actuated microstructures considering nonlinear effects", Commun. Nonlinear Sci. Numer. Simul. 14 (2009), 1664-1678.

[Mojahedi et al. 2010] M. Mojahedi, M. Moghimi Zand, and M. T. Ahmadian, "Static pull-in analysis of electrostatically actuated microbeams using homotopy perturbation method", Appl. Math. Model. 34 (2010), 1032-1041.

[Papargyri-Beskou et al. 2003b] S. Papargyri-Beskou, K. G. Tsepoura, D. Polyzos, and D. E. Beskos, "Bending and stability analysis of gradient elastic beams", Int. J. Solids Struct. 40 (2003b), 385-400.

[Park and Gao 2006] S. K. Park and X. L. Gao, "Bernoulli-Euler beam model based on a modified couple stress theory", $J$. Micromech. Microeng. 16 (2006), 2355-2359.

[Reddy 2003] J. N. Reddy, Mechanics of laminated composite plates and shells: theory and analysis, 2nd ed., CRC Press, Boca Raton, FL, 2003.

[Reddy 2007] J. N. Reddy, Theory and analysis of elastic plates and shells, 2nd ed., CRC Press, Boca Raton, FL, 2007.

[Scheible et al. 2002] D. V. Scheible, A. Erbe, and R. H. Blick, "Evidence of a nanomechanical resonator being driven into chaotic response via the Ruelle-Takens route", Appl. Phys. Lett. 81 (2002), 1884-1886.

[Timoshenko and Goodier 1970] S. P. Timoshenko and J. N. Goodier, Theory of elasticity, 3rd ed., McGraw-Hill, New York, 1970.

[Toupin 1962] R. A. Toupin, "Elastic materials with couple-stresses”, Arch. Ration. Mech. Anal. 11 (1962), 385-414. 
[Tsepoura et al. 2002] K. Tsepoura, S. Papargyri-Beskou, D. Polyzos, and D. E. Beskos, "Static and dynamic analysis of a gradient-elastic bar in tension", Arch. Appl. Mech. 72 (2002), 483-497.

[Turner et al. 1998] K. L. Turner, S. A. Miller, P. G. Hartwell, N. C. MacDonald, S. H. Strogatz, and S. G. Adams, "Five parametric resonances in a microelectromechanical system", Nature 396 (1998), 149-152.

[Vardoulakis and Sulem 1995] I. Vardoulakis and J. Sulem, Bifurcation analysis in geomechanics, Blackie/Chapman \& Hall, London, 1995.

[Wang et al. 2010] B. Wang, J. Zhao, and S. Zhou, "A micro scale Timoshenko beam model based on strain gradient elasticity theory", Eur. J. Mech. A. Solids 29 (2010), 591-599.

[Yang et al. 2002] F. Yang, A. C. M. Chong, D. C. C. Lam, and P. Tong, "Couple stress based strain gradient theory for elasticity", Int. J. Solids Struct. 39 (2002), 2731-2743.

Received 7 Sep 2011. Revised 2 Feb 2012. Accepted 17 Feb 2012.

REZA ANSARI: $r_{-}$ansari@guilan.ac.ir

Department of Mechanical Engineering, University of Guilan, P.O. Box 3756, Rasht, Iran

RAHEB GHOLAMI: rahebgholami@gmail.com

Department of Mechanical Engineering, University of Guilan, P.O. Box 3756, Rasht, Iran

Mohammad Ali DarabI: mad.fluid.darabi@gmail.com

Department of Mechanical Engineering, University of Guilan, P.O. Box 3756, Rasht, Iran 


\title{
JOURNAL OF MECHANICS OF MATERIALS AND STRUCTURES
}

\author{
jomms.net
}

\author{
Founded by Charles R. Steele and Marie-Louise Steele \\ EDITORS \\ Charles R. STEele \\ DAVIDE BIGONI \\ Stanford University, USA \\ University of Trento, Italy \\ IWONA JASIUK University of Illinois at Urbana-Champaign, USA \\ YASUHIDE SHINDO Tohoku University, Japan
}

\section{EDITORIAL BOARD}

H. D. Bui École Polytechnique, France

J. P. CARTER University of Sydney, Australia

R. M. CHRISTENSEN Stanford University, USA

G. M. L. GLadWELL University of Waterloo, Canada

D. H. Hodges Georgia Institute of Technology, USA

J. HUTCHINSON Harvard University, USA

C. HwU National Cheng Kung University, Taiwan

B. L. KARIHALOO University of Wales, UK

Y. Y. KIM Seoul National University, Republic of Korea

Z. Mroz Academy of Science, Poland

D. PAmplona Universidade Católica do Rio de Janeiro, Brazil

M. B. RUBIN Technion, Haifa, Israel

A. N. SHUPIKOV Ukrainian Academy of Sciences, Ukraine

T. TARNAI University Budapest, Hungary

F. Y. M. WAN University of California, Irvine, USA

P. WRIGGERS Universität Hannover, Germany

W. YANG Tsinghua University, China

F. ZIEGLER Technische Universität Wien, Austria

PRODUCTIONｃontact@msp.org

Silvio LEVY Scientific Editor

See http://jomms.net for submission guidelines.

JoMMS (ISSN 1559-3959) is published in 10 issues a year. The subscription price for 2012 is US $\$ 555 /$ year for the electronic version, and \$735/year (+\$60 shipping outside the US) for print and electronic. Subscriptions, requests for back issues, and changes of address should be sent to Mathematical Sciences Publishers, Department of Mathematics, University of California, Berkeley, CA 94720-3840.

JoMMS peer-review and production is managed by EditFLOw ${ }^{\circledR}$ from Mathematical Sciences Publishers.

\author{
mathematical sciences publishers \\ http://msp.org/ \\ A NON-PROFIT CORPORATION \\ Typeset in $\mathrm{IAT}_{\mathrm{E}} \mathrm{X}$ \\ Copyright $@ 2012$ by Mathematical Sciences Publishers
}




\title{
Journal of Mechanics of Materials and Structures
}

\author{
Volume 7, No.2 February 2012
}

Micromechanical analysis of unidirectional composites using a least-squares-based differential quadrature element method

Mohammad Bayat and Mohammad Mohammadi Aghdam

Size-dependent free vibration analysis of infinite nanotubes using elasticity theory

JAFAR ESKandari JaM, YASER Mirzaei, Behnam GHeShlaghi

and ReZa AvaZmohammadi

Spectral element model for the vibration of a spinning Timoshenko shaft

USIK LEE and INJOON JANG

On indenter boundary effects at elastic contact

Denis Jelagin and Per-Lennart LaRsson 165

Reflection of $P$ and $S V$ waves from the free surface of a two-temperature thermoelastic solid half-space

BALJEET SingH and KirAN BALA

A nonlinear Timoshenko beam formulation based on strain gradient theory ReZa Ansari, RAHEb GHOLAmi and MOHAMMAD ALI DARABi

Finite element analysis of bending-stiff composite conical shells with multiple delamination 